\title{
Painel em Madeira de Reflorestamento e Chapas de Partículas para Instalações Rurais
}

\author{
Diogo de Lucca Sartori ${ }^{1}$, Julio Cesar Machado Cravo ${ }^{1}$, \\ Nubia Garzon Barrero ${ }^{1}$, Juliano Fiorelli², Holmer Savastano Junior ${ }^{2}$ \\ ${ }^{1}$ Programa de Pós-Graduação em Zootecnia, Faculdade de Zootecnia e Engenharia de Alimentos - FZEA, \\ Universidade de São Paulo - USP \\ ${ }^{2}$ Departamento de Engenharia de Alimentos, Faculdade de Zootecnia e Engenharia de Alimentos - FZEA, \\ Universidade de São Paulo - USP
}

\begin{abstract}
RESUMO
O trabalho teve como objetivo desenvolver painel em madeira de reflorestamento e chapas de partículas de bagaço de cana-de-açúcar e resina poliuretana bicomponente à base de óleo de mamona, como alternativa para o sistema convencional de fechamento lateral do tronco coletivo de centro de manejo para bovinos de corte. A caracterização físico-mecânica do material forneceu um indicativo das possibilidades de uso para esse produto, que associado a pilares roliços de eucalipto compõem o sistema modular estrutural. A avaliação da resistência ao impacto do sistema modular, que procurou simular o efeito provocado pelo animal, em condições de uso, demonstrou a eficiência do modelo estrutural proposto para uso em centro de manejo.
\end{abstract}

Palavras-chave: bagaço de cana, resina poliuretana, centro de manejo.

\section{Panel in Reforestation Wood and Particleboards for Rural Facilities}

\begin{abstract}
The objective of this study was to develop a panel in reforestation wood and particleboards of sugarcane bagasse and bi-component polyurethane resin on castor oil basis, as an alternative for the conventional system of side closing of the working chute in cattle handling facilities. The physical-mechanical characterization of the material provided an indication of the possible uses for this product, that associated with eucalyptus rounded pillars compose the structural modular system. The impact resistance evaluation of the modular system, which tried to simulate the effect produced by the animals under working condition, demonstrated the efficiency of the structural model proposed for use in cattle handling facilities.
\end{abstract}

Keywords: sugarcane bagasse, polyurethane resin, cattle handling facilities 


\section{INTRODUÇÃO}

Detentor do maior rebanho comercial bovino do mundo, com 176,6 milhões de cabeças (Anualpec, 2010), o agronegócio brasileiro possui a pecuária de corte como uma das mais importantes atividades, representando uma parcela substancial do PIB e gerando mais de 9 milhões de empregos diretos e indiretos. A partir da década de 90 , com as imposições da globalização, o setor pecuário (corte) tem apresentado avanços expressivos de desenvolvimento, aumentando sua competitividade produtiva e econômica. Firmando-se como o maior exportador mundial de carne bovina, o Brasil vem conquistando cada vez mais mercados por todo o mundo.

Atualmente o País ocupa o terceiro lugar no consumo mundial de carne bovina e o segundo em produção de equivalente de carcaça, 7.778 milhares de $t$ (Anualpec, 2010). Com uma taxa de abate de $23 \%$, representado por 41,2 milhões de cabeças por ano. No ano de 2010, o Brasil exportou uma quantia de $1.230 .571 \mathrm{t}$ de carne, o que representa quase 5 bilhões de dólares.

Inicialmente, a produção de gado de corte não possui muita tecnologia agregada. De maneira geral é extensiva, à base de pasto, exigindo maior área em comparação com a bovinocultura de leite, por exemplo. A produção é anual, requer menos cuidados quando comparada à exploração leiteira. O rebanho abrange maior número de cabeças e, além disso, exige capital inicial maior, apesar das instalações serem mais simples e rústicas, quase sempre se resumindo em centro de manejo e piquetes (Souza et al., 2003).

No entanto, com o avanço da necessidade da implantação de novas tecnologias, devido ao aumento da exigência do setor, a busca por instalações mais adequadas e que atendam às novas exigências do mercado estão sendo solicitadas e, muitas vezes, implantadas pelos produtores rurais. Sendo assim, o desenvolvimento de painel de partícula à base de bagaço de cana-de-açúcar e resina poliuretana bicomponente à base de óleo de mamona, além de representar um produto sustentável, poderá substituir com eficiência as tábuas de madeira utilizadas em fechamento de seringas e de tronco coletivo de centro de manejo para bovino de corte.
Existem diferentes tipos de sistemas de produção de bovinos de corte (extensivo, semi-intensivo e confinado), sendo que, em todos esses, o centro de manejo (ou curral de manejo, curral de manobra, mangueira) são necessários e indispensáveis, independente do tipo de sistema de criação.

Um centro de manejo compreende um grupo de estruturas necessárias ao manejo de bovinos de corte, que entre elas estão os curraletes, brete, tronco, balança e embarcadouro ou carregador. É usado para confinar seguramente e eficientemente os bovinos para observação, manejo sanitário de rotina e procedimentos de manejo.

O brete conduz os animais da seringa para o curralete ou tronco. Sua finalidade é manter os animais em uma fila única para que possam entrar no tratamento ou área de carga um de cada vez. $\mathrm{O}$ brete deve ter pelo menos 6 metros de comprimento, independente do tamanho do rebanho. As laterais do brete devem ser sólidas, como as da seringa, para prevenir que os animais vejam as pessoas, equipamentos e outras distrações do lado de fora, assim mantendo-os calmos. A seringa é usada para afunilar os animais para o brete, sendo que um projeto adequado de seringa diminui o trabalho necessário com o gado. $\mathrm{O}$ tamanho da seringa deve ser para manejar oito a dez animais ao mesmo tempo (Bicudo et al., 2002).

Segundo Huhnke \& Harp (2009), o brete e a seringa são considerados o coração do curral, tendo como algumas características desejáveis para o tronco coletivo: ser curvado, laterais totalmente fechadas e laterais inclinadas. Já para a seringa, as principais características desejáveis seriam: forma circular e laterais totalmente fechadas.

Os painéis aglomerados surgiram na Alemanha, no início da década de 40, como forma de viabilizar a utilização de resíduos de madeira, em face da dificuldade de obtenção de madeiras de boa qualidade para produção de lâminas para compensados, devido ao isolamento do país durante a segunda Guerra Mundial (Mendes et al., 2010). Esses painéis são geralmente fabricados a partir de partículas de madeira aglutinadas por adesivo sintético ou outro aglomerante, sendo o conjunto prensado a quente, por tempo suficiente para que a cura da resina se efetue (Iwakiri et al., 2004). 
Esses painéis são geralmente fabricados a partir de partículas de madeira aglutinadas por adesivo sintético ou outro aglomerante, sendo o conjunto prensado a quente, por tempo suficiente para que a cura da resina se efetue (Iwakiri et al., 2004; Hillig, 2000).

Segundo Maciel et al. (2004), no Brasil, a madeira para produção de painéis aglomerados vem de florestas plantadas e de empresas produtoras e, entre estas, algumas utilizam madeira de Pinus na sua linha de produção, outras empregam apenas Eucalipto e algumas combinam Pinus e Eucalipto em proporções variáveis.

A princípio, esses painéis também podem ser fabricados a partir de qualquer outro material lignocelulósico que lhes confiram alta resistência mecânica e peso específico pré-estabelecido, já que a composição química dos materiais lignocelulósicos é semelhante à da madeira, mais precisamente com a das madeiras duras que contêm menor teor de lignina e maior teor de hemiceluloses do tipo pentosanas (Rowell et al., 2000).

A produção de painéis de partículas de resíduos lignocelulósicos é uma alternativa que proporciona agregar valor a esse subproduto, possibilitando atender à crescente demanda da indústria de painéis de madeira, além de contribuir com a diminuição do uso da madeira e consequentemente a pressão sobre as florestas, reduzindo custos de produção dos painéis, tornando-os mais competitivos no cenário econômico (Mendes et al., 2010).

Para a produção de painéis de partículas, o bagaço de cana-de-açúcar está entre os materiais mais promissores dentre outros resíduos agroindustriais, devido ao seu baixo custo e abundância. A estrutura lignocelulósica do bagaço é semelhante à da madeira (Santana \& Teixeira, 1993), mais precisamente com a das madeiras duras que contêm menor teor de lignina e maior teor de pentosanas (Sousa et al., 1986).

O bagaço de cana-de-açúcar, que é um conjunto de fibras emaranhadas de celulose e subproduto da agroindústria, está disponível em grande quantidade devido ao aumento da área plantada, decorrente principalmente de investimentos públicos e privados na produção alcooleira e da industrialização do processo de produção de açúcar e álcool. Segundo dados da CONAB (2011), a safra de cana-de-açúcar no Brasil no ano de 2010 foi de aproximadamente 603 milhões de $t$, o que representa para o País geração de cerca de 168 milhões $t$ de bagaço de cana, pois, de acordo com Silva et al. (2007), cada tonelada processada de cana-de-açúcar gera um total de $280 \mathrm{~kg}$ de resíduo.

Geralmente, o bagaço de cana é queimado em reservatórios de vapor para produzir energia para uso industrial, mas, atualmente, devido às suas características físico-químicas, está sendo utilizado em um vasto campo de pesquisas, dentre os quais, na produção de ração animal, indústria química para a produção de plástico biodegradável, na produção de bicarbonato de sódio e cloreto de amônio, e também para a manufatura de painéis aglomerados (Silva, et al., 2007; Anselmi, 2007).

As projeções de produção de bagaço de cana demonstram um aumento contínuo ao longo dos anos, com uma estimativa de produção de 285 milhões t para a safra 2020/2021, de acordo com CONAB (2011).

Quanto às propriedades e qualidade dos painéis, muitos são os fatores que contribuem para as características do produto final, dentre eles pode-se destacar: a densidade do material lignocelulósico, densidade dos painéis, umidade das partículas, razão de compactação, ciclo de prensagem, quantidade e tipo de adesivo (Kelly, 1977; Maloney, 1993).

Vários trabalhos foram desenvolvidos visando avaliar a viabilidade de uso de resíduos lignocelulósicos na produção de painéis de partículas, Barros Filho (2009) trabalhou com desenvolvimento e caracterização de painéis aglomerados à base de cana-de-açúcar e resinas ureia formaldeído e melamina formaldeído, obteve resultados próximos ou superiores aos encontrados na literatura para painéis à base de bagaço de cana-de-açúcar.

José \& Beraldo (2006) utilizaram partículas de Bambusa vulgaris Schrad e, como aglomerante, resina poliuretana à base de óleo de mamona, com a finalidade de buscar sustentabilidade para o processo produtivo. Pelos resultados obtidos, pode-se afirmar que a relação custo-benefício, tendo em vista que a resina é o material de maior custo econômico e ambiental na composição da chapa, torna-se mais viável a produção de chapas com $10 \%$ de resina. 
Dentro desse escopo, o presente trabalho teve como objetivo desenvolver um sistema modular estrutural em madeira de reflorestamento e painéis de partículas de bagaço de cana-de-açúcar e resina poliuretana bicomponente à base de óleo de mamona, como alternativa para o sistema convencional de fechamento lateral do tronco coletivo (brete) de centro de manejo para bovinos de corte.

\section{MATERIAL E MÉTODOS}

Os painéis de partículas de bagaço de cana-deaçúcar e resina poliuretana bicomponente à base de óleo de mamona e o protótipo do sistema modular estrutural foram produzidos no Laboratório de Construções e Ambiência, FZEA/USP-Pirassununga.

O bagaço de cana-de-açúcar, para a produção dos painéis de partícula, foi obtido em usina sucroalcooleira localizada na região de PirassunungaSP. Antes de receber qualquer processamento, foi secado em estufa a $60{ }^{\circ} \mathrm{C}$, sendo posteriormente peneirado para a retirada da porção mais fina. A obtenção das partículas ocorreu por meio da moagem em moinho de facas com malha de $8 \mathrm{~mm}$.

Para a adesão das partículas, foi utilizada resina poliuretana bicomponente à base de óleo de mamona, sendo um dos componentes poliol derivado do óleo vegetal, e o outro o isocianato polifuncional.

Para a confecção do protótipo do sistema modular estrutural, foram utilizadas vigas de madeira do gênero Eucalyptus, provenientes de áreas de reflorestamento.

Os painéis de partículas tiveram como parâmetros de fabricação prensagem a $5 \mathrm{MPa}$ em um tempo de 10 minutos, temperatura a $100{ }^{\circ} \mathrm{C}$ e densidade nominal de $1,0 \mathrm{~g} / \mathrm{cm}^{3}$. As dimensões dos painéis produzidos foram de $520 \times 520 \times 20 \mathrm{~mm}$ para os painéis utilizados no protótipo e $400 \times 400 \times 10 \mathrm{~mm}$ para painéis submetidos à caracterização físicomecânica (Figura 1 e 2).

Para o protótipo, foram utilizados quatro painéis fixados na estrutura de madeira de eucalipto com parafusos, formando uma peça com dimensões de 2,20 de comprimento e 52 de largura (Figuras 3 e 4). Essas dimensões foram estipuladas com base naquelas utilizadas em centro de manejo de bovinos

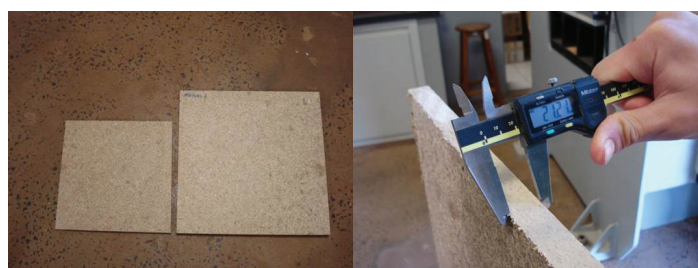

Figura 1. Painéis de partículas de bagaço de cana-deaçúcar.

Figure 1. Particleboards of sugarcane bagasse.

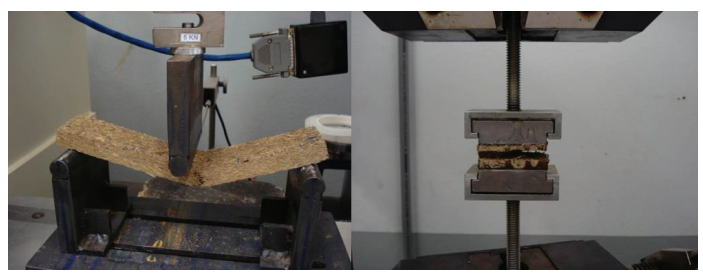

Figura 2. Ensaio de flexão e tração perpendicular. Figure 2. Bending test and internal bond test.

de corte, procurando um modelo estrutural próximo do real.

Depois da prensagem, os painéis permaneceram 72 horas em processo de estabilização, para posterior esquadrejamento e corte dos corpos de prova (CP), seguindo as recomendações da norma ABNT (2006) NBR 14810:2006 - Chapas de madeira aglomerada. Os painéis que foram usados no protótipo apenas foram esquadrejados para a uniformização das dimensões.

Foram realizados ensaios para propriedades físicas: densidade, inchamento e absorção de água (2h e $24 \mathrm{~h}$ ); e mecânicas: módulo de ruptura (MOR), módulo de elasticidade (MOE) e tração paralela (TP) de acordo com a norma ABNT (2006) NBR 14810:2006.

$\mathrm{O}$ inchamento consiste na diferença de espessura antes e depois da imersão dos $\mathrm{CP}$ em água em um período de 2 e 24 horas. As medições foram feitas usando um paquímetro digital com exatidão de $0,1 \mathrm{~mm}$. Os ensaios de inchamento fornecem indicações a respeito das condições de adesão e de resistência das partículas que constituem o painel quando submetidos à imersão em água. Esses ensaios são realizados sistematicamente pelas indústrias, para controle de qualidade de seus produtos.

Absorção de água consiste na diferença de peso antes e depois da imersão dos $\mathrm{CP}$ em água em 

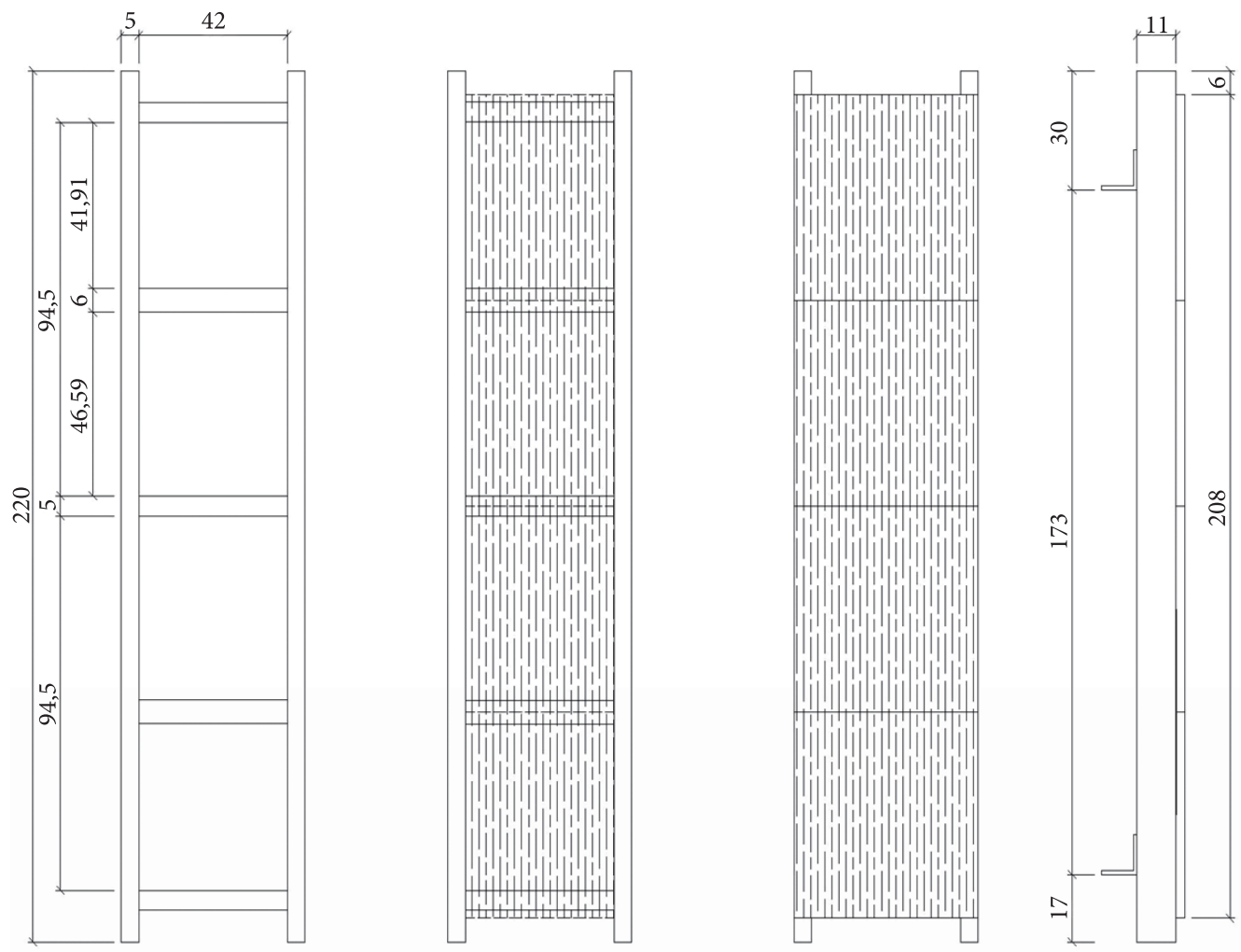

Figura 3. Desenho do protótipo com suas dimensões.

Figure 3. Design of the prototype with their dimensions.

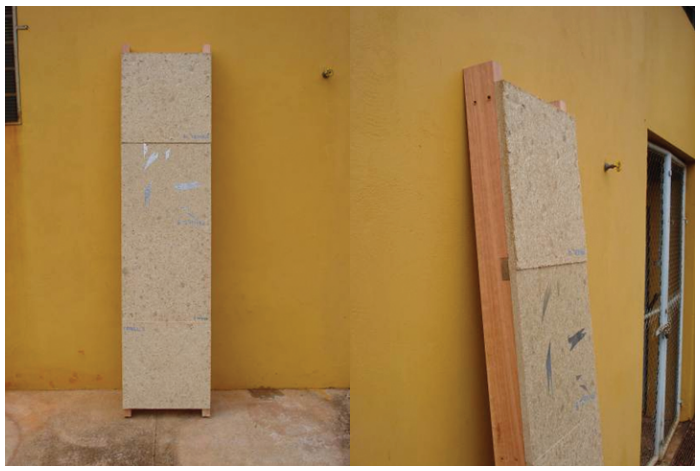

Figura 4. Ilustração do protótipo estrutural em madeira e painéis de partículas.

Figure 4. Illustration of the structural prototype in wood and particleboards.

um período de 2 e 24 horas, usando uma balança semianalítica, com resolução de $0,1 \mathrm{~g}$.

O ensaio de tração perpendicular e flexão foram realizados utilizando uma máquina universal de ensaios em condições de laboratório. A capacidade de carga para o ensaio de tração perpendicular foi de $4 \mathrm{~mm} / \mathrm{min}$. Módulo de ruptura (MOR) e módulo de elasticidade (MOE) foram determinados a 3 pontos, operando com uma célula de carga de $5 \mathrm{kN}$ e velocidade de $6 \mathrm{~mm} / \mathrm{min}$. A distância entre pontos foi de $200 \mathrm{~mm}$.

Com a finalidade de se comprovar a eficiência do sistema modular estrutural, foi realizado ensaio de impacto de corpo mole seguindo preceitos da normativa ABNT (1990) MB-3256:1990 - Divisórias leves internas moduladas - Verificação da resistência a impactos, e ABNT (2008) NBR 15575:2008 - Edifícios habitacionais de até cinco pavimentos - Desempenho. Como parâmetros para o ensaio, foram aplicadas no protótipo diferentes intensidades de forças (energia) e aferido o deslocamento sofrido pelo componente construtivo. A Tabela 1 demonstra as intensidades de forças e energias correspondentes. Sabendo que o peso de um bovino é em média equivalente a $500 \mathrm{~kg}$, o valor da energia aplicada, que simulasse o impacto do animal no modelo, foi de até $960 \mathrm{~J}$. 


\section{RESULTADOS E DISCUSSÃO}

Os resultados das propriedades físico-mecânicas obtidos foram comparados com os indicados pela norma ANSI A208.1:1999 - mat-formed wood particleboard, e NBR 14810:2006 (ABNT, 2006), para painéis aglomerados de madeira. Seus usos se justificaram pela semelhança do produto desenvolvido no presente estudo com um painel de partículas de madeira.

A Tabela 2 apresenta resultados das propriedades físicas dos painéis em estudo e valores mínimos recomendados pelos documentos normativos. Foi obtido resultado de $947 \mathrm{~kg} / \mathrm{m}^{3}$ para densidade, indicando e classificando os painéis como sendo de alta densidade, segundo a Norma ANSI A208.1:1999 e acima do preconizado pela norma NBR 14810:2006 (ABNT, 2006), sendo um valor entre 551 e $750 \mathrm{~kg} /$ $\mathrm{m}^{3}$. Essa classificação é importante, pois valores mínimos de módulo de elasticidade (MOE), módulo de ruptura (MOR), tração perpendicular (TP), inchamento e absorção guardam estreita relação com a densidade. Valores semelhantes foram obtidos por Marcilio et al. (2008), que avaliaram as propriedades

Tabela 1. Altura e energia de cada impacto correspondente ao saco de areia.

Table 1. Height and energy corresponding to the impact of each bag of sand.

\begin{tabular}{|cc|}
\hline $\mathbf{h}(\mathbf{m})$ & $\mathbf{E}(\mathbf{J})$ \\
\hline 0,15 & 60 \\
0,3 & 120 \\
\hline 0,45 & 180 \\
\hline 0,6 & 240 \\
0,9 & 360 \\
\hline 1,2 & 480 \\
\hline 1,8 & 720 \\
\hline 2,4 & 960 \\
\hline
\end{tabular}

físicas de painéis à base de bagaço da cana-de-açúcar e fibras das folhas caulinares do bambu (palha de bambu), da espécie Dendrocalamus giganteus e resina ureia e obtiveram painéis com densidade de até $1 \mathrm{~g} / \mathrm{cm}^{3}$.

Estes resultados foram superiores aos $0,6 \mathrm{~g} / \mathrm{cm}^{3}$ obtidos por Battistelle et al. (2009), em painéis à base de bagaço de cana e resina ureia.

Os painéis avaliados apresentaram valores de inchamento em 2 horas, abaixo dos $8 \%$ recomendados pela norma NBR 14810:2006 (ABNT, 2006), mas valores a cima do recomendado pela norma A208.1:1999 em 24 horas.

A Tabela 3 apresenta valores de propriedades mecânicas dos painéis em estudo e valores mínimos recomendados pelos documentos normativos. Em relação ao módulo de ruptura (MOR), verificase que o valor médio de 22,67 $\mathrm{MPa}$ está dentro do recomendado para painéis de alta densidade pela norma A208.1:1999 e superior à recomendada pela norma NBR 14810:2006 (ABNT, 2006). Sendo assim, é possível classificar os painéis em estudo como sendo de alta densidade e indicá-los para uso industrial e comercial.

O valor de MOR do painel em estudo foi superior aos obtidos por Contreras et al. (2006), Silva et al. (2008), Battistelle et al. (2009), Okino et al. (1997) e Widyorini et al. (2005), demonstrando que o processo de produção utilizado foi consistente e que a resina bicomponente à base de óleo de mamona se apresenta como potencial adesivo para fabricação de painéis de partículas de bagaço de cana-de-açúcar.

O valor do módulo de elasticidade (MOE) dos painéis em estudo foi superior ao recomendado pela norma A208.1:1999, que é de $2750 \mathrm{MPa}$.

Os resultados obtidos para MOE mostram-se superiores àqueles encontrados por Okino et al.

Tabela 2. Valores médios das propriedades físicas.

Table 2. Mean values of physical properties.

\begin{tabular}{lcccc}
\multicolumn{2}{c}{ Propriedades físicas } & Experimental & NBR 14810 & ANSI A208.1 \\
Densidade $\left(\mathrm{kg} / \mathrm{m}^{3}\right)$ & & $947(5,51)$ & $551-750$ & 800 \\
Inchamento (\%) & 2 hours & $5,8(20,9)$ & 8 & - \\
& 24 hours & $20,0(13,8)$ & - & - \\
\multirow{2}{*}{ Absorção (\%) } & 2 hours & $5,6(8,8)$ & - & - \\
\hline
\end{tabular}


Tabela 3. Valores médios das propriedades mecânicas.

Table 3. Mean values of mechanical properties.

\begin{tabular}{cccc} 
Propriedades mecânicas & Experimental & NBR 14810 & ANSI A208.1 \\
\hline MOE $(\mathrm{MPa})$ & $2848(7,29)$ & - & $2400-2750$ \\
MOR $(\mathrm{MPa})$ & $22,60(6,32)$ & 18 & $16,5-23,5$ \\
\hline TP $(\mathrm{MPa})$ & $1,18(31,64)$ & 0,40 & $0,9-1,0$ \\
\hline
\end{tabular}

(1997), que identificaram valores de MOE abaixo de $2300 \mathrm{MPa}$ para painéis aglomerados à base de bagaço de cana-de-açúcar e resina ureia.

Em relação à tração perpendicular às fibras, os painéis em estudo apresentaram valores superiores àqueles recomendados pelos documentos normativos NBR 14810:2006 (ABNT, 2006), e A208.1:1999 e também acima de $0,4 \mathrm{MPa}$, valor encontrado por Battistelle et al. (2009) e de 0,6 MPa, obtido por Okino et al. (1997). Estes resultados demonstram que o nível de resina utilizado está adequado, sendo possível avaliar a possibilidade de redução de adesivo com o objetivo de economia no processo de fabricação (José \& Beraldo, 2006).

Para o ensaio de corpo mole, que avaliou a resistência ao impacto do sistema modular, simulando o efeito provocado pelo animal, em condições de uso, foi observado que o protótipo em estudo apresentou deslocamentos horizontais aceitáveis, dentro dos limites estabelecidos pela norma. Sendo, portanto, indicado para uso em centro de manejo de bovinos, desde que respeitadas às condições de projeto.

\section{CONCLUSÕES}

Com base nas condições em que este trabalho foi realizado, podem-se inferir as seguintes conclusões:

- A resina poliuretana à base de óleo de mamona mostra-se como alternativa eficiente para produção de painéis de partículas à base de bagaço de cana-de-açúcar, em substituição à resina ureiaformaldeído empregada atualmente pela indústria;

- Parâmetros de produção dos painéis estão adequados, comprovado pelos resultados físicomecânicos;

- É possível fabricar painéis de partículas de bagaço de cana, com valores de propriedades mecânicas que atendem às normas e se equiparam aos das chapas fabricadas em escala industrial, à base de partículas de madeira; e

- O resultado obtido através do ensaio de impacto, mostrou que o sistema modular estrutural em madeira de reflorestamento e painéis de partículas é indicado para o uso em centro de manejo para bovinos.

\section{AGRADECIMENTOS}

Os autores agradecem à FAPESP o apoio concedido à pesquisa.

\section{STATUS DA SUBMISSÃO}

Recebido: 16/09/2011

Aceito: 07/02/2012

Resumo publicado online: 22/03/2012

Artigo completo publicado: 30/06/2012

\section{AUTOR(ES) PARA CORRESPONDÊNCIA}

\section{Diogo de Lucca Sartori}

Laboratório de Construções e Ambiência, Faculdade de Zootecnia e Engenharia de Alimentos - FZEA, Universidade de São Paulo - USP, Av. Duque de Caxias Norte, 225, Campus da USP, CEP 13635-900, Pirassununga, SP e-mail: diogosartori@usp.br

\section{REFERENNCIAS}

American National Standards Institute - ANSI. ANSI A208: 1: Mat-formed wood particleboard: Specification. Gaithersburg; 1999.

Anselmi R. Diversificação industrial inclui bioeletricidade, levedura, plástico biodegradável e gás carbônico. Jornalcana 2007; 58.

ANUALPEC. Anuário da Agropecuária Brasileira. São Paulo: FNP Consultoria e Comércio; 2010. p. 5. 
Associação Brasileira de Normas Técnicas - ABNT. MB-3256: Divisórias leves internas moduladas. Rio de Janeiro: ABNT; 1990.

Associação Brasileira de Normas Técnicas - ABNT. NBR 14810: Chapas de madeira aglomerada. Rio de Janeiro: ABNT; 2006.

Associação Brasileira de Normas Técnicas - ABNT. NBR 15575: edifícios habitacionais de até cinco pavimentos. Rio de Janeiro: ABNT; 2008.

Barros Filho RM. Painéis aglomerados a base de canade-açúcar e resinas uréia formaldeído e melamina formaldeído [dissertação]. Ouro Preto: Universidade Federal de Ouro Preto; 2009.

Battistelle RAG, Marcilio C, Lahr FAR. Emprego do bagaço da cana-de-açúcar (Saccharum officinarum) e das folhas caulinares do bambu da espécie Dendrocalamus giganteus na produção de chapas de partículas. Revista Minerva 2009; 5(3):297-305.

Bicudo JR, Mcneill S, Turner LW, Burris R, Anderson J. Cattle handling facilities: Planning, components and layouts. Lexington: University of Kentucky Cooperative Extension Service; 2002.

Companhia Nacional de Abastecimento - CONAB. Acompanhamento de safra Brasileira: cana-de-açúcar, primeiro levantamento.. Brasília: CONAB; 2011.

Contreras W, Owen M, Ballester VC, Contreras Y, Garay D. Diseño de Tableros de Partículas de Caña Brava y Adhesivo Fenol - formaldehído. Revista Forestal Latinoamericana 2006; 39:39-55.

Hillig E. Qualidade de chapas aglomeradas estruturais, fabricadas com madeiras de Pinus, Eucalipto e Acácia negra, puras ou misturadas, coladas com taninoformaldeido [dissertação]. Santa Maria: Universidade Federal de Santa Maria; 2000.

Huhnke RL, Harp S. Corral and Working Facilities for Beef Cattle. Stillwater, Oklahoma: Oklahoma State University, Cooperative Extension Service; 2009.

Iwakiri S, Shimizu J, Silva JC, Menessi CHSD, Puehringher A, Venson I et al. Produção de painéis de madeira aglomerada de Grevillea robusta A. Cunn. ex R. Br. Revista Árvore 2004; 28(6):883-887. http://dx.doi. org/10.1590/S0100-67622004000600013

José, FJ, Beraldo, AL. Chapas prensadas de partículas de bambu e adesivo poliuretana à base de óleo de mamona. In: Anais do X Encontro Brasileiro em Madeiras e em Estruturas de Madeira; 2006; São Pedro. São Paulo; 2006. v. 1, p.1-11.

Kelly MW. Critical literature review of relationships between processing parameters and physical properties of particleboard. USDA Forest Service, Forest Products Laboratory General; 1977. Technical Report FPL-10.

Maciel AS, Vital BR, Della Lucia RMD, Pimenta AS. Painéis de partículas aglomeradas de madeira de Pinus elliottii Elgelm., poliestireno (PS) e poliestireno tereftalato (PET). Revista Árvore 2004; 28(2):257-266. http://dx.doi.org/10.1590/S0100-67622004000200012

Maloney TM. Modern particleboard and dry-process fiberboard manufacturing. 2nd ed. São Francisco: M. Freeman; 1993.

Marcilio C, Battistelle RAG, Valarelli ID. Fabricação de chapas de partículas compostas de bagaço de canade-açúcar e fibras das folhas caulinares do bambu: caracterização física e mecânica. In. Anais do XI Encontro Brasileiro em Madeira e Estruturas de Madeira; 2008; Londrina. São Paulo; 2008. CD-ROOM.

Mendes RF, Mendes LM, Abranches RAS, Dos Santos RC, Guimarães Júnior JB. Painéis aglomerados produzidos com bagaço de cana em associação com madeira de eucalipto. Scientia Forestalis 2010; 38(86):285-295.

Okino EYA, Andahur JPV, Santana MAE, Souza MR. Physico-mechanical properties of chemically modified sugar cane bagasse partideboards. Scientia Forestalis 1997; 52:35-42.

Rowell RM, Han JS, Rowell JS. Characterization and Factors Affecting Fiber Properties. In: Anais do Natural Polymers and Agrofibers Based Composites; 2000; São Carlos. São Carlos: Embrapa Instrumentação Agropecuária Editora.

Santana MAE, Teixeira DE. Uso de bagaço de canade-açúcar na confecção de chapas aglomeradas. In: Anais do VII Congresso Florestal Brasileiro; I Congresso Florestal Panamericano; 1993; Curitiba. Curitiba: SBS/ SBEF, 1993. p. 667-672.

Silva VLMM, Gomes WCO, Alsina LS. Utilização do bagaço de cana de açúcar como biomassa adsorvente na adsorção de poluentes orgânicos. Revista Eletrônica de Materiais e Processos 2007; 2:27-32.

Sousa MFB, Schuchardt U, Rodrigues JAR. Separação e identificação dos constituintes do bagaço de cana-deaçúcar pelo processo "Organosolv". Ciência e cultura 1986; 38(1):181-188.

Souza CF, Tinoco IFF, Sartor V. Informações básicas para projetos de construções rurais. Viçosa; 2003.

Widyorini R, Xu J, Umemura K, Kawai S. Manufacture and properties of binderless particleboard from bagasse I: effects of raw material type, storange methods and manufacturing process. Journal of Wood Science 2005; 51(6):648-654 